\title{
ON THE EXISTENCE AND UNIQUENESS OF SOLUTIONS OF THE CAUCHY PROBLEM FOR WAVE EQUATIONS WITH GENERAL STOCHASTIC MEASURES
}

UDC 519.21

\author{
D. M. GORODNYA
}

\begin{abstract}
The existence and uniqueness of a solution of the Cauchy problem for wave equations containing a term expressed via the integral with respect to a stochastic measure are proved. Some general properties of generalized functions assuming values in a Fréchet space are used in the proof of the uniqueness of a solution.
\end{abstract}

\section{INTRODUCTION}

Stochastic wave equations describe the physical process of propagation of waves under some random influences. The random influences in various mathematical models are usually described in terms of stochastic integrals where the integrators are supposed to satisfy certain conditions of regularity or to possess some moments, for example, the stochastic term expressed via an integral with respect to a Wiener process in [1] or with respect to a martingale measure in [2, 3. In the paper 4], the stochastic term is determined by a process with Hölder trajectories. In the current paper, the stochastic influence is described by an integral with respect to a stochastic measure being $\sigma$-additive in probability.

Mild solutions of the Cauchy problem for a wave equation involving stochastic measures are considered in [5]. A solution of such an equation is defined in the case of $d$ spatial variables as a process $V_{t}, t>0$, such that, for each $t>0$ fixed, the function $V_{t}$ is a generalized random function defined in the space $D\left(\mathbb{R}^{d}\right)$ of test functions in $C^{\infty}\left(\mathbb{R}^{d}\right)$ that has a compact support and satisfies, in a certain sense, the corresponding equation and initial condition. In the current paper, a solution of the wave equation for the case of $d$ spatial variables is understood as a generalized random function defined in $D\left(\mathbb{R}^{d+1}\right)$. This allows us to prove the existence and uniqueness of a solution under assumptions similar to those used in [6] where the existence and uniqueness of a generalized solution for usual wave equations are obtained.

Some applications of wave equations with stochastic measures are given in [5] (also see the references therein). Mild solutions of the Cauchy problem for the wave equation with a stochastic measure are studied in [7] for the case of a single spatial variable.

\section{Preliminary Results}

Let $(\Omega, F, \mathrm{P})$ be a complete probability space and let $L_{0}$ be a family of all classes of $\mathrm{P}$-equivalent random variables in $(\Omega, F, \mathrm{P})$. The convergence in $L_{0}$ is understood in the sense of the convergence in probability.

2010 Mathematics Subject Classification. Primary 60G57, 60H15, 60H05, 35L05, 46F99.

Key words and phrases. Stochastic wave equation, Cauchy problem, stochastic measure, Fréchet space, generalized function. 
Definition 2.1 ([5]). Any linear continuous mapping $\xi: D\left(\mathbb{R}^{d+1}\right) \rightarrow L_{0}$ is called a generalized random function.

The family of all generalized random functions is denoted by $D_{r}^{\prime}\left(\mathbb{R}^{d+1}\right)$.

Let $\mathbf{B}=\mathbf{B}\left(\mathbb{R}^{d+1}\right)$ be the $\sigma$-algebra of Borel sets in the space $\mathbb{R}^{d+1}$.

Definition $2.2([5])$. Any $\sigma$-additive mapping $\mu: \mathbf{B} \rightarrow L_{0}$ is called a stochastic measure.

The integrals $\int_{A} f d \mu$ are defined and studied in the paper [8] for the case where $A \in \mathbf{B}$ and $f$ is a measurable real valued function. In particular, an analogue of the Lebesgue dominated convergence theorem is proved in [8] (see Corollary 1.2 in [8]; also see Proposition 7.1.1 in [9]) and a result on the differentiability of the integral with respect to a parameter is obtained in [5]. Every stochastic measure $\mu$ determines a generalized random function $\dot{\mu}$ according to the rule

$$
(\dot{\mu}, \varphi)=\int_{\mathbb{R}^{d+1}} \varphi(x, t) d \mu(x, t), \quad \varphi \in D\left(\mathbb{R}^{d+1}\right) .
$$

Note that the derivative of a stochastic process $\eta:(a ; b) \rightarrow L_{0}$ is defined for the convergence in probability and that an arbitrary bounded measurable function is integrable with respect to every stochastic measure.

\section{Existence of a solution of the Cauchy Problem}

Consider the Cauchy problem for the wave equation that can be written as follows:

$$
\frac{\partial^{2} V}{\partial t^{2}}=a^{2} \triangle_{x} V+\dot{\mu}, \quad t>0,\left.\quad \frac{\partial V}{\partial t}\right|_{t=0+}=\dot{\varsigma},\left.\quad V\right|_{t=0+}=\dot{\nu},
$$

where $V \in D_{r}^{\prime}\left(\mathbb{R}^{d+1}\right)$ is an unknown function. Here $a>0, \mu$ is a stochastic measure in $\mathbf{B}\left(\mathbb{R}^{d+1}\right)$ vanishing at all measurable subsets of $K:=\left\{(x, t) \in \mathbb{R}^{d+1}: t<0\right\}$, and where $\varsigma$ and $\nu$ are stochastic measures in $\mathbf{B}\left(\mathbb{R}^{d}\right)$.

Now we give the rigorous definition of a solution.

Definition 3.1. A generalized random function $V \in D_{r}^{\prime}\left(\mathbb{R}^{d+1}\right)$ is called a solution of the Cauchy problem (3.1) if $V=0$ for $t<0$ and if

$$
\begin{aligned}
\left(V, \frac{\partial^{2} \varphi(x, t)}{\partial t^{2}}\right)= & a^{2}\left(V, \triangle_{x} \varphi(x, t)\right)+\int_{\mathbb{R}^{d+1}} \varphi(x, t) d \mu(x, t)-\int_{\mathbb{R}^{d}} \frac{\partial \varphi(x, 0)}{\partial t} d \varsigma(x) \\
& +\int_{\mathbb{R}^{d}} \varphi(x, 0) d \nu(x)
\end{aligned}
$$

for all $\varphi \in D\left(\mathbb{R}^{d+1}\right)$.

Similarly to [6, $\S 5.5]$, we have $V=0$ for $t<0$ if $(V, \varphi)=0$ for all $\varphi \in D\left(\mathbb{R}^{d+1}\right)$ such that supp $\varphi \subset K$.

A solution of the Cauchy problem (3.1) in the case of $d=1$ is constructed according to the d'Alembert formula [6, §13.4] as explained below. For $\varphi \in D\left(\mathbb{R}^{2}\right)$, put

$$
\begin{gathered}
r_{1}(x, t, \varphi):=\frac{1}{2 a} \int_{t}^{\infty} d s \int_{x-a(s-t)}^{x+a(s-t)} \varphi(y, s) d y ; \\
r_{3}(x, \varphi):=r_{1}(x, 0, \varphi) ; \quad r_{2}(x, \varphi):=r_{3}\left(x, \frac{\partial \varphi}{\partial s}\right) .
\end{gathered}
$$

Theorem 3.1. A solution of the Cauchy problem (3.1) for $d=1$ is a generalized random function such that

$$
(V, \varphi)=\int_{\mathbb{R} \times[0 ; \infty)} r_{1}(x, t, \varphi) d \mu(x, t)-\int_{\mathbb{R}} r_{2}(x, \varphi) d \varsigma(x)+\int_{\mathbb{R}} r_{3}(x, \varphi) d \nu(x)
$$


for $\varphi \in D\left(\mathbb{R}^{2}\right)$.

Proof. Fix $\varphi \in D\left(\mathbb{R}^{2}\right)$ and $(x, t) \in \mathbb{R} \times[0 ; \infty)$. Since $\varphi$ has a bounded support, there exists $L>0$ such that the support of $\varphi$ belongs to $(-L ; L)^{2}$. Thus

$$
r_{1}(x, t, \varphi)=\frac{1}{2} \int_{0}^{L} d \tau \int_{-1}^{1} \varphi(x+a \tau \xi, t+\tau) d \xi .
$$

If $\varphi$ is fixed, the latter equality implies that the function $r_{1}$ is bounded and continuous with respect to the variables $x$ and $t$ belonging to the set $\mathbb{R} \times[0 ; \infty)$. Therefore $r_{1}$ is an integrable function with respect to the stochastic measure $\mu$.

If $(x, t) \in \mathbb{R} \times[0 ; \infty)$ is fixed, then

$$
\begin{aligned}
\varphi(x, t) & =(\delta(y-x, s-t), \varphi(y, s))=\left(\mathcal{E}_{1}(y-x, s-t), \frac{\partial^{2} \varphi(y, s)}{\partial s^{2}}-a^{2} \frac{\partial^{2} \varphi(y, s)}{\partial y^{2}}\right) \\
& =\frac{1}{2 a} \int_{t}^{\infty} d s \int_{x-a(s-t)}^{x+a(s-t)}\left(\frac{\partial^{2} \varphi(y, s)}{\partial s^{2}}-a^{2} \frac{\partial^{2} \varphi(y, s)}{\partial y^{2}}\right) d y
\end{aligned}
$$

where $\delta$ and $\mathcal{E}_{1}$ denote the Dirack $\delta$ function and the fundamental solution of the wave operator for $d=1$, respectively (see [6]). Hence

$$
\begin{aligned}
\int_{\mathbb{R} \times[0 ; \infty)} \varphi(x, t) d \mu(x, t)= & \int_{\mathbb{R} \times[0 ; \infty)} r_{1}\left(x, t, \frac{\partial^{2} \varphi}{\partial s^{2}}\right) d \mu(x, t) \\
& -a^{2} \int_{\mathbb{R} \times[0 ; \infty)} r_{1}\left(x, t, \frac{\partial^{2} \varphi}{\partial y^{2}}\right) d \mu(x, t),
\end{aligned}
$$

whence we conclude that the generalized random function $\int_{\mathbb{R} \times[0 ; \infty)} r_{1}(x, t, \varphi) d \mu(x, t)$, $\varphi \in D\left(\mathbb{R}^{2}\right)$, is a solution of the Cauchy problem

$$
\frac{\partial^{2} V}{\partial t^{2}}=a^{2} \triangle_{x} V+\dot{\mu}, \quad t>0,\left.\quad \frac{\partial V}{\partial t}\right|_{t=0+}=\left.V\right|_{t=0+}=0 .
$$

Using equalities (3.3) for $t=0$ and inclusions $\frac{\partial \varphi}{\partial t} \in D\left(\mathbb{R}^{2}\right)$, we check similarly to (3.4) that the generalized random functions $\int_{\mathbb{R}} r_{3}(x, \varphi) d \nu, \varphi \in D\left(\mathbb{R}^{2}\right)$, and $\int_{\mathbb{R}} r_{2}(x, \varphi) d \varsigma$, $\varphi \in D\left(\mathbb{R}^{2}\right)$, are solutions of the Cauchy problems

$$
\frac{\partial^{2} V}{\partial t^{2}}=a^{2} \triangle_{x} V, \quad t>0,\left.\quad \frac{\partial V}{\partial t}\right|_{t=0+}=0,\left.\quad V\right|_{t=0+}=\dot{\nu}
$$

and

$$
\frac{\partial^{2} V}{\partial t^{2}}=a^{2} \triangle_{x} V, \quad t>0,\left.\quad \frac{\partial V}{\partial t}\right|_{t=0+}=\dot{\varsigma},\left.\quad V\right|_{t=0+}=0,
$$

respectively.

It remains to note that the sum of solutions of problems (3.4), (3.5), and (3.6) is a solution of the Cauchy problem (3.1) for $d=1$.

If $d=2$, then the Poisson formula [6, \$13.4] is used to construct a solution of the Cauchy problem (3.1). In what follows we use the notation introduced in [6]. Namely, let $U(x, r):=\left\{x \in \mathbb{R}^{2}:|x| \leq r\right\}$. For $\varphi \in D\left(\mathbb{R}^{3}\right)$, put

$$
\begin{aligned}
v_{1}(x, t, \varphi) & :=\frac{1}{2 \pi a} \int_{t}^{\infty} d s \int_{U(x, a(s-t))} \frac{\varphi(y, s) d y}{\sqrt{a^{2}(s-t)^{2}-|y-x|^{2}}} \\
v_{3}(x, \varphi) & :=v_{1}(x, 0, \varphi) ; \quad v_{2}(x, \varphi):=v_{3}\left(x, \frac{\partial \varphi}{\partial s}\right) .
\end{aligned}
$$


Theorem 3.2. If $d=2$, then a solution of the Cauchy problem (3.1) is a generalized random function such that

$$
(V, \varphi)=\int_{\mathbb{R}^{2} \times[0 ; \infty)} v_{1}(x, t, \varphi) d \mu(x, t)-\int_{\mathbb{R}^{2}} v_{2}(x, \varphi) d \varsigma(x)+\int_{\mathbb{R}^{2}} v_{3}(x, \varphi) d \nu(x)
$$

for $\varphi \in D\left(\mathbb{R}^{3}\right)$.

A solution of the Cauchy problem (3.1) for $d=3$ can be constructed with the help of the Kirchhoff formula [6, \$13.4] as follows. Let $S(x, r):=\left\{x \in \mathbb{R}^{3}:|x|=r\right\}$. For $\varphi \in D\left(\mathbb{R}^{4}\right)$, put

$$
\begin{gathered}
w_{1}(x, t, \varphi):=\frac{1}{4 \pi a^{2}} \int_{t}^{\infty} d \tau\left(\frac{1}{\tau-t} \int_{S(x, a(\tau-t))} \varphi(y, \tau) d S_{y}\right) \\
w_{3}(x, \varphi):=w_{1}(x, 0, \varphi) ; \quad w_{2}(x, \varphi):=w_{3}\left(x, \frac{\partial \varphi}{\partial \tau}\right) .
\end{gathered}
$$

Theorem 3.3. A solution of the Cauchy problem (3.1) for $d=3$ is a generalized random function such that

$$
(V, \varphi)=\int_{\mathbb{R}^{3} \times[0 ; \infty)} w_{1}(x, t, \varphi) d \mu(x, t)-\int_{\mathbb{R}^{3}} w_{2}(x, \varphi) d \varsigma(x)+\int_{\mathbb{R}^{3}} w_{3}(x, \varphi) d \nu(x)
$$

for $\varphi \in D\left(\mathbb{R}^{4}\right)$.

The proofs of Theorems 3.2 and 3.3 follow the lines of the proof of Theorem 3.1. Thus we omit those proofs.

\section{Generalized functions in Fréchet spaces}

Below we define and study generalized functions assuming values in a Fréchet space. In what follows we use their properties to prove the uniqueness of a solution of the Cauchy problem (3.1).

We adapt the terminology and definition of [10, Section I.9]. A linear space $X$ equipped with a quasinorm $\|\cdot\|$ is called a Fréchet space if $X$ is complete with respect to the metric $\rho(x, y)=\|x-y\|, x, y \in X$. In particular, the space $L_{0}$ with the quasinorm defined above is a Fréchet space. The convergence with respect to the quasinorm in this space is equivalent to the convergence in probability [8, $\S 0.2]$.

Let $X$ be a Fréchet space equipped with a quasinorm $\|\cdot\|$. Denote the zero element in $X$ by $\overline{0}$.

Definition 4.1. Any linear continuous mapping $F: D\left(\mathbb{R}^{d}\right) \rightarrow X$ is called an $X$-generalized function.

In what follows $D_{X}^{\prime}\left(\mathbb{R}^{d}\right), D^{\prime}\left(\mathbb{R}^{d}\right)$, and $\langle F, \varphi\rangle,(f, \varphi)$ denote the space of all $X$-generalized functions, standard space of generalized functions [6, §5.3], and actions of the function $F \in D_{X}^{\prime}\left(\mathbb{R}^{d}\right)$ and $f \in D^{\prime}\left(\mathbb{R}^{d}\right)$ at a test function $\varphi \in D\left(\mathbb{R}^{d}\right)$, respectively.

For $F \in D_{X}^{\prime}\left(\mathbb{R}^{d}\right)$, we use the standard notation supp $F$ for the support of $F$. The equality $F=\overline{0}$ in an open set of $\mathbb{R}^{d}$, linear change, multiplication by a function $\alpha \in C^{\infty}\left(\mathbb{R}^{d}\right)$, and the derivative are defined similarly to the case of $D^{\prime}\left(\mathbb{R}^{d}\right)$ (see, for example, [6]). The convolution $F * g$ for functions $F \in D_{X}^{\prime}\left(\mathbb{R}^{d}\right)$ and $g \in D^{\prime}\left(\mathbb{R}^{d}\right)$ is defined by

$$
\langle F * g, \varphi\rangle:=\lim _{k \rightarrow \infty}\left\langle F(x),\left(g(y), \eta_{k}(x, y) \varphi(x+y)\right)\right\rangle, \quad \varphi \in D\left(\mathbb{R}^{d}\right) .
$$

Here $\left\{\eta_{k}\right\}$ is an arbitrary sequence of functions belonging to $D\left(\mathbb{R}^{2 d}\right)$ and converging to 1 in $\mathbb{R}^{2 d}$ in the sense explained in [6, $\left.\S 7.4\right]$. 
Similarly to the case of usual generalized functions, we say that $F * g$ exists if the above limit exists for every $\varphi \in D\left(\mathbb{R}^{d}\right)$ and does not depend on a sequence $\left\{\eta_{k}\right\}$. The inclusion $F * g \in D_{X}^{\prime}\left(\mathbb{R}^{d}\right)$ is checked by using the same method as in the case of usual generalized functions.

We use the following well-known properties of linearity and differentiability for the convolution to prove the main results below.

1) Linearity. If the convolutions $F_{1} * g, F_{2} * g, F * g_{1}$, and $F * g_{2}$ exist, then the convolutions

$$
\left(\lambda F_{1}+\gamma F_{2}\right) * g=\lambda\left(F_{1} * g\right)+\gamma\left(F_{2} * g\right)
$$

and

$$
F *\left(\lambda g_{1}+\gamma g_{2}\right)=\lambda\left(F * g_{1}\right)+\gamma\left(F * g_{2}\right)
$$

exist for all numbers $\lambda$ and $\gamma$.

2) Differentiability. If the convolution $F * g$ exists, then the convolutions $\mathbb{D}^{\beta} F * g$ and $F * \mathbb{D}^{\beta} g$ exist for all $\beta=\left(\beta_{1}, \ldots, \beta_{d}\right)$ with $\beta_{j} \in \mathbb{N} \cup\{0\}, 1 \leq j \leq d$; moreover

$$
\mathbb{D}^{\beta}(F * g)=\mathbb{D}^{\beta} F * g=F * \mathbb{D}^{\beta} g .
$$

The proof of the property of linearity of the convolution is trivial. The general case of the property of differentiability follows from its particular case for first derivatives $\mathbb{D}_{j}$, $1 \leq j \leq d$.

Fix $j$ such that $1 \leq j \leq d, \varphi \in D\left(\mathbb{R}^{d}\right)$, and a sequence $\left\{\eta_{k}\right\} \subset \mathbb{R}^{2 d}$ converges to 1 in $\mathbb{R}^{2 d}$. Then the sequence of functions $\left\{\eta_{k}+\frac{\partial \eta_{k}}{\partial x_{k}}\right\}$ converges to 1 in $\mathbb{R}^{2 d}$. Taking into account the lemma on the derivative of a test function of a special form proved in 6 , $\S 7.1]$ we obtain

$$
\begin{aligned}
\left\langle\mathbb{D}_{j}(F * g), \varphi\right\rangle= & -\lim _{k \rightarrow \infty}\left\langle F(x),\left(g(y), \eta_{k}(x, y) \frac{\partial \varphi(x+y)}{\partial x_{j}}\right)\right\rangle \\
= & -\lim _{k \rightarrow \infty}\left\langle F(x),\left(g(y), \frac{\partial}{\partial x_{j}}\left(\eta_{k}(x, y) \varphi(x+y)\right)\right)\right\rangle \\
& +\lim _{k \rightarrow \infty}\left\langle F(x),\left(g(y),\left(\eta_{k}(x, y)+\frac{\partial \eta_{k}(x, y)}{\partial x_{j}}\right) \varphi(x+y)\right)\right\rangle \\
& -\lim _{k \rightarrow \infty}\left\langle F(x),\left(g(y), \eta_{k}(x, y) \varphi(x+y)\right)\right\rangle \\
= & -\lim _{k \rightarrow \infty}\left\langle F(x), \mathbb{D}_{j}\left(g(y), \eta_{k}(x, y) \varphi(x+y)\right)\right\rangle \\
= & \left\langle\mathbb{D}_{j} F(x),\left(g(y), \eta_{k}(x, y) \varphi(x+y)\right)\right\rangle .
\end{aligned}
$$

Thus the convolution $\mathbb{D}_{j} F * g$ exists and the equality $\mathbb{D}_{j} F * g=\mathbb{D}_{j}(F * g)$ holds. Note also that

whence

$$
\frac{\partial \varphi(x+y)}{\partial x_{j}}=\frac{\partial \varphi(x+y)}{\partial y_{j}},
$$

$$
\begin{aligned}
\left\langle\mathbb{D}_{j}(F * g), \varphi\right\rangle= & -\lim _{k \rightarrow \infty}\left\langle F(x),\left(g(y), \eta_{k}(x, y) \frac{\partial \varphi(x+y)}{\partial y_{j}}\right)\right\rangle \\
= & -\lim _{k \rightarrow \infty}\left\langle F(x),\left(g(y), \frac{\partial}{\partial y_{j}}\left(\eta_{k}(x, y) \varphi(x+y)\right)\right)\right\rangle \\
& +\lim _{k \rightarrow \infty}\left\langle F(x),\left(g(y),\left(\eta_{k}(x, y)+\frac{\partial \eta_{k}(x, y)}{\partial y_{j}}\right) \varphi(x+y)\right)\right\rangle \\
& -\lim _{k \rightarrow \infty}\left\langle F(x),\left(g(y), \eta_{k}(x, y) \varphi(x+y)\right)\right\rangle \\
= & -\lim _{k \rightarrow \infty}\left\langle F(x),\left(\mathbb{D}_{j} g(y), \eta_{k}(x, y) \varphi(x+y)\right)\right\rangle .
\end{aligned}
$$


This implies that the convolution $F * \mathbb{D}_{j} g$ exists and the equality $F * \mathbb{D}_{j} g=\mathbb{D}_{j}(F * g)$ holds. Therefore the property of differentiability is proved.

Let $L(\mathbb{D})=\sum_{|\alpha|=0}^{m} a_{\alpha} \mathbb{D}^{\alpha}$ be a differential operator with constant coefficients and let $\mathcal{E}$ be the fundamental solution of the operator $L(\mathbb{D})$ (see, for example, [6, §11.2]).

Theorem 4.1. Let the convolution $F * \mathcal{E}$ exist for a function $F \in D_{X}^{\prime}\left(\mathbb{R}^{d}\right)$. Then the equation $L(\mathbb{D}) U=F$ has a solution in $D_{X}^{\prime}\left(\mathbb{R}^{d}\right)$. This solution can be written as $U=F * \mathcal{E}$ and is unique in the class of all functions belonging to $D_{X}^{\prime}\left(\mathbb{R}^{d}\right)$ for which the convolution with $\mathcal{E}$ exists.

The proof of Theorem 4.1 is the same as that in the case of usual generalized functions; see [6, §11.3]. In doing so, one should use the equality $F * \delta=F$ for all $F \in D_{X}^{\prime}\left(\mathbb{R}^{d}\right)$.

Theorem 4.2. Let two functions $\mathcal{F} \in D_{X}^{\prime}\left(\mathbb{R}^{d+1}\right)$ and $h \in D^{\prime}\left(\mathbb{R}^{d+1}\right)$ be such that

1) $\mathcal{F}=\overline{0}$ for $t<0$;

2) $\operatorname{supp} h$ belongs to the set

$$
\bar{\Gamma}_{+}(d)=\left\{(x, t) \in \mathbb{R}^{d+1}: \text { at }-|x| \geq 0\right\} .
$$

Then the convolution $\mathcal{F} * h$ exists; moreover $\mathcal{F} * h=\overline{0}$ for $t<0$.

To prove Theorem 4.2 we use the same reasoning as in the case of usual generalized functions; see [6, §12.2].

\section{Uniqueness of a SOlution of the CAUChy PRoblem for a WAVE EQUATion}

Theorem 5.1. The Cauchy problem (3.1) has a unique solution if $d=1,2,3$.

Proof. Put $X=L_{0}$. According to Definition 3.1, the generalized random function $V$ is a solution of the Cauchy problem (3.1) if and only if $V$ is a solution of the equation $\square_{a} V=G$ in $D_{X}^{\prime}\left(\mathbb{R}^{d+1}\right)$ and if $V=0$ for $t<0$. Here

$$
\square_{a}:=\frac{\partial^{2}}{\partial t^{2}}-a^{2} \triangle_{x}
$$

is the wave operator and $G$ is a given $X$-generalized function defined by

$$
(G, \varphi)=\int_{\mathbb{R}^{d+1}} \varphi(x, t) d \mu(x, t)-\int_{\mathbb{R}^{d}} \frac{\partial \varphi(x, 0)}{\partial t} d \varsigma(x)+\int_{\mathbb{R}^{d}} \varphi(x, 0) d \nu(x) .
$$

Since the stochastic measure $\mu$ vanishes for all measurable subsets of the set $K$, we conclude that $G=0$ for $t<0$. Furthermore, if $d=1,2,3$, then the support of the fundamental solution $\mathcal{E}_{d}$ of the wave operator $\square_{a}$ belongs to the set $\bar{\Gamma}_{+}(d)$. Thus the convolution $G * \mathcal{E}_{d}$ exists in view of Theorem 4.2. Now the uniqueness of the solution of the Cauchy problem follows from Theorem 4.1.

\section{BIBLIOGRAPHY}

1. A. Millet and P. Morien, On a non linear stochastic wave equation in the plane: existence and uniqueness of the solution, Ann. Appl. Probab. 11 (2007), 922-951.

2. R. C. Dalang, Extending martingale measure stochastic integral with applications to spatially homogeneous S.P.D.E's, Electronic J. Probab. 4 (1999), no. 6, 1-29. MR1684157(2000b:60132)

3. R. C. Dalang and N. E. Frangos, The stochastic wave equation in two spatial dimensions, Ann. Probab. 26 (1998), no. 1, 187-212. MR.1617046 (99c:60127)

4. L. Quer-Sardanyons and S. Tindel, The 1-d stochastic wave equation driven by a fractional Brownian sheet, Stoch. Prosses. Appl. 17 (2007), 1448-1472. MR2353035 (2008j:60152)

5. V. N. Radchenko, Heat equation and wave equation with general stochastic measures, Ukr. Matem. Zh. 60 (2008), no. 12, 1675-1685; English transl. in Ukrain. Math. J. 60 (2008), 19681981. MR 2523115 (2010d:60117)

6. V. S. Vladimirov, Equations of Mathematical Physics, "Nauka", Moscow, 1981; English transl., "Mir", Moscow, 1979. MR653331 (83i:00029) 
7. I. Bodnarchuk, Mild solution of the wave equation with a general random measure, Visnyk Kyiv Nats. Univer. Mathematics. Mechanics 24 (2010), 28-33. (Ukrainian)

8. V. N. Radchenko, Integrals With Respect to General Random Measures, Institute of Mathematics, National Academy of Science of Ukraine, Kyiv, 1999. (Russian)

9. S. Kwapień and W. A. Woycziński, Random Series and Stochastic Integrals: Single and Multiple, Birkhäuser, Boston, 1992. MR1167198 (94k:60074)

10. K. Yosida, Functional Analysis, Springer, Berlin, 1965.

Department of Mathematical Analysis, Faculty for Mechanics and Mathematics, National Taras Shevchenko University, Academician Glushkov Avenue, 4e, Kiev 03127, Ukraine

E-mail address: gorodnyaya@yandex.ru

Received 23/JUNE/2011

Translated by S. KVASKO 